\title{
Perturbation Effect on IIR Filters
}

\author{
Shweta Pathania and Ajmer Singh
}

Department of electronics and communication, Lovely Professional University, Jalandhar-Delhi G.T. Road, National Highway 1, Phagwara, Punjab-144411, India; shwetapathania3099@gmail.com, ajmersinghbhinder@gmail.com

\section{Abstract}

During the realization of digital FIR and IIR filters the finite word length in computers affects the accuracy of filter coefficients. This leads to change in actual desired poles and zeros of the system function. In this paper firstly the analog filter is converted to digital domain using bilinear transformation and then the filter coefficients are quantized to analyze the perturbation error and the finite word length effect.This paper analyzes the sensitivity to quantization of filter coefficients for 8-bit, 16-bit and 32-bit precisions up to order 7 for digital low pass Butterworth, Chebyshev (type I and type II) and Elliptic filters for direct form realization. In the end result shows that the Chebyshev type II filter yields the least perturbation error and Elliptic filter yields the maximum perturbation error for higher orders.

Keywords: Bilinear Transformation, Jacobian Elliptic Function, Perturbation Error

\section{Introduction}

The numbers are represented using binary digits 0 or 1 in computers. This leads to signal quantization and analog to digital conversion noise. The finite word length arithmetic needed for processing the numbers is implemented using two different approaches, depending upon the ease of implementation and the accuracy as well as dynamic range needed in processing. The digital IIR filter realization increases the pole sensitivity problem. The pole zero locations, filter responses and stability gets affectedwhen the filter coefficients are quantized. General IIR filter is described by

$H(z)=\frac{\sum_{k=0}^{M} b_{k} z^{-k}}{1+\sum_{k=1}^{N} a_{k} z^{-k}}$

In Eq. $1 a_{k}$ is quantized to $\hat{a}_{k}$ then the new filter system function is represented as

$\hat{H}(z)=\frac{\sum_{k=0}^{M} b_{k} z^{-k}}{1+\sum_{k=1}^{N} \hat{a}_{k} z^{-k}}$

This new filter defined in Eq. 2 is different from the original $H(z)$. These filters can be compared on various aspects like magnitude responses, phase responses, or change in their pole zero locations. MATLAB is a powerful tool to investigate these changes and their overall effect on the usability of the filter. The total perturbation error $\Delta p_{m}$ is expressed in the form

$$
\boldsymbol{\Delta} p_{m}=-\sum_{k=\mathbf{1}}^{N} \frac{p_{m}^{N-k}}{\prod_{\substack{l=\mathbf{1} \\ l \neq m}}^{N}\left(p_{m}-p_{l}\right)} \boldsymbol{\Delta} a_{k}
$$

This Eq. 3 shows the measure of the movement of the $m$ th pole $p_{m}$ to changes in the coefficients $\left\{a_{k}\right\}$. It is clear from the expression that the perturbation error increases if the term $\left(p_{m}-p_{l}\right)$ is very small as having inverse relationship. The coefficients $\left\{a_{k}\right\}$ of Eq. 1 are quantized to coefficients $\left\{\hat{a}_{k}\right\}$ after the conversion of analog filter into digital domain using bilinear transformation in MATLAB. Similarly the numerator coefficients $\left\{b_{k}\right\}$ can be quantized to coefficients $\left\{\hat{b}_{k}\right\}$ and further the sensitivity of zeros to changes in the parameters $\left\{b_{k}\right\}$ can also be realized To investigate the effect of coefficient quantization on filter behavior, all the filter coefficients $\left\{a_{k}\right\}$ are represented using the same number of integer and fraction bits. The function is implemented on MATLAB using rounding operation on sign magnitude format. Then the perturbation error as given by Eq. 3 is calculated for the IIR filters for direct form realization.

\section{Digital Butterworth Filter}

The maximally flat magnitude squared frequency response 
of analog low pass Butterworth filter is characterized as

$|\mathrm{H}(\mathrm{j} \omega)|^{2}=\frac{1}{1+\left(\omega / \omega_{\mathrm{c}}\right)^{2 M}}$

The frequency response defined by Eq. 4 is converted in digital domain using MATLAB function $[b, a]=\operatorname{butter}\left(n, W_{n}\right)$. Figure $1(\mathrm{a})$ to $1(\mathrm{~g})$ shows the pole-zero plot for infinite precision, from Figure 1(h) to Figure 1(n) for 8-bit precision, Figure 1(o) to Figure $1(\mathrm{v})$ for 16 bit precision and Figure 1(w) to Figure 1(ab) for 32 bit precision coefficients $\mathbb{\{}\left\{a \rrbracket_{k}\right\}$ for digital low pass Butterworth filter from order 1 to 7 respectively. The variation in pole zero locations are computed up to order 7 for direct form realization. In Figurel when the bit precision is small the pole location varies at a greater extent as order of the filter increases.

Table 1. Perturbation error for digital

Butterworth filter for 8-bit, 16-bit and 32-bit

precision

\begin{tabular}{lccc}
\hline Order & 8-bit & 16-bit & 32-bit \\
\hline 1 & 0 & 0 & 0 \\
2 & $7.1198 \mathrm{e}-204$ & $1.0068 \mathrm{e}-205$ & $3.0902 \mathrm{e}-211$ \\
3 & $5.1348 \mathrm{e}-200$ & $1.6073 \mathrm{e}-202$ & $3.0606 \mathrm{e}-207$ \\
4 & $2.3916 \mathrm{e}-192$ & $7.4186 \mathrm{e}-195$ & $1.1311 \mathrm{e}-199$ \\
5 & $1.3306 \mathrm{e}-177$ & $1.6098 \mathrm{e}-179$ & $3.0112 \mathrm{e}-184$ \\
6 & $2.5518 \mathrm{e}-152$ & $7.7717 \mathrm{e}-155$ & $2.3752 \mathrm{e}-159$ \\
7 & $1.5882 \mathrm{e}-144$ & $2.9453 \mathrm{e}-119$ & $6.3047 \mathrm{e}-122$ \\
\hline
\end{tabular}

When $W_{n}=1$ and $n$ varies from 1 to 7 the total perturbation error $\boldsymbol{\Delta} p_{m}$ calculated for the digital Butterworth filter is as shown in Table 1.

\section{Digital Chebyshev Filter}

\subsection{Digital Chebyshev Type I}

Chebyshev Type I filters have a magnitude response that ripples in the pass band and monotonic in stop band $\mathrm{d}^{5}$. The magnitude squared frequency response of analoglow passChebyshev type I filter is given as

$|H(j \omega)|^{2}=\frac{1}{1+\varepsilon^{2} P_{M}^{2}\left(\omega / \omega_{p}\right)}$

where $\varepsilon$ is a constant related to ripples in pass band, $\omega_{p}$ is the pass band frequency and $P_{M}\left(\omega / \omega_{p}\right)$ is a polynomial in Eq. 5 is defined as

$P_{M}\left(\omega / \omega_{p}\right)= \begin{cases}\cos \left[M \cos ^{-1}-\left(\omega / \omega_{p}\right)\right], & \left.|\omega| \leq \omega_{p}\right] \\ \cosh \left[M \cosh ^{-1}-\left(\omega / \omega_{p}\right)\right], & |\omega|] \leq \omega_{p}\end{cases}$

In Eq. 5 and Eq. $6 \mathrm{Mis}$ the order of the filter. The analog Chebyshev type I low pass filter is converted to digital domain using the MATLAB inbuilt function

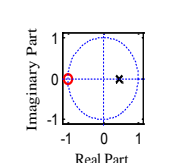

Real Part

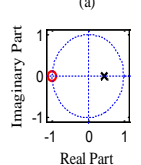

Real Part
(h)

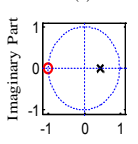

Real Part
(0)

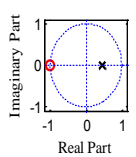

Real Part
(v)

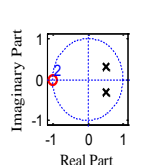

Real Part
(b)

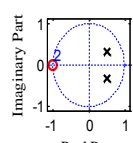

Real Part

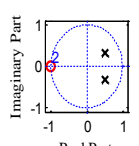

Real Part
(p)

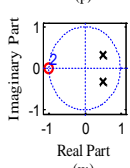

Real Part

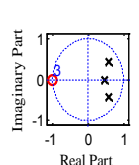

Real Part
(c)
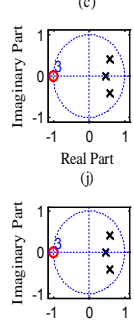

Real Part
(q)

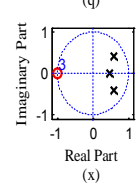

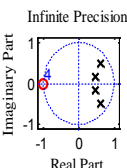

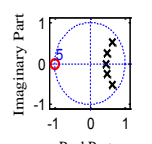

Real Part
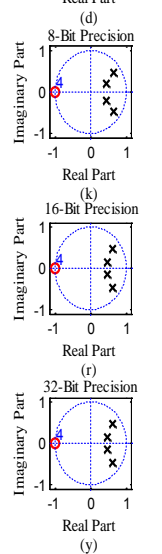
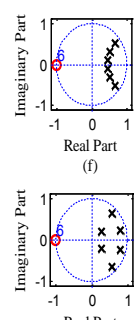

Real Part

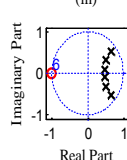

Real Part

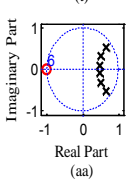

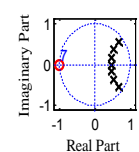

Real Part
(g)

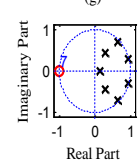

Real Part

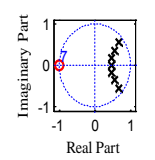

(u)

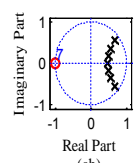

(ab)

Figure 1. Pole and zero plot of digital butterworth filter for infinite, 8-bit, 16-bit and 32-bit precision, where red circle indicates the zeros and black cross indicates the poles of the filter. 
$[b, a]=\operatorname{cheby} 1\left(n, R, W_{p}\right)$. Figure $2(\mathrm{a})$ to $2(\mathrm{~g})$ shows the pole-zero plot for infinite precision, from Figure 2(h) to Figure 2(n) for 8-bit precision, Figure 2(o) to Figure $2(\mathrm{v})$ for 16 bit precision and Figure 2(w) to Figure 2(ab) for 32 bit precision coefficients $\mathbb{Z}\left\{a \rrbracket_{k}\right\}$ for digital low pass Chebyshev type I filter from order 1 to 7 respectively.

In Figure 2(g), Figure 2(m) and Figure 2(n) polezero plots of 8 bit precision shows that the filter becomes unstable for higher order and less precision.

When $R=2 d B, W_{p}=1$ and $n$ is the order that varies from 1 to 7 , the total perturbation error $\Delta p_{m}$ is calculated for the digital Chebyshev type I filter is as shown in Table 2.

Table 2. Perturbation error for digital chebyshev type I filter for 8-bit, 16-bit and 32-bit precision

\begin{tabular}{lccc}
\hline Order & 8-bit & 16-bit & 32-bit \\
\hline 1 & 0 & 0 & 0 \\
2 & $5.6597 \mathrm{e}-205$ & $2.9601 \mathrm{e}-207$ & $4.2261 \mathrm{e}-213$ \\
3 & $3.2923 \mathrm{e}-202$ & $2.2272 \mathrm{e}-204$ & $1.7908 \mathrm{e}-209$ \\
4 & $3.4686 \mathrm{e}-197$ & $1.3062 \mathrm{e}-199$ & $1.0040 \mathrm{e}-204$ \\
5 & $4.5975 \mathrm{e}-187$ & $2.6303 \mathrm{e}-189$ & $3.5915 \mathrm{e}-194$ \\
6 & $7.8115 \mathrm{e}-170$ & $9.1632 \mathrm{e}-172$ & $1.7811 \mathrm{e}-176$ \\
7 & $5.9458 \mathrm{e}-143$ & $1.8493 \mathrm{e}-146$ & $1.3123 \mathrm{e}-150$ \\
\hline
\end{tabular}

\subsection{Digital Chebyshev Type II}

The magnitude response of Chebyshev type II filter have ripples in stop band and monotonic in pass band.

The magnitude squared frequency response of analog low pass Chebyshev type II filter is given as

$|H(j \omega)|^{2}=\frac{\varepsilon^{2} P_{M}^{2}\left(\omega_{s} / \omega\right)}{1+\varepsilon^{2} P_{M}^{2}\left(\omega_{s} / \omega\right)}$

where $\varepsilon$ is a constant related to rinoles in stop band, $\omega_{s}$ is the stop band frequency and $P_{M}\left(\omega / \omega_{p}\right)$ in Eq. 8 is a polynomial defined as

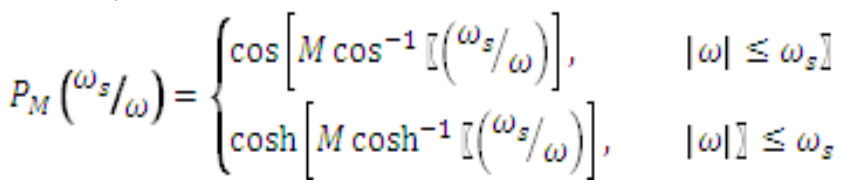

Figure 3(a) to Figure 3(g) shows the pole-zero plot for infinite precision, from Figure 3(h) to Figure 3(n) for 8-bit precision, Figure 3(o) to Figure 3(v) for 16 bit precision and Figure $3(\mathrm{w})$ to Figure $3(\mathrm{ab})$ for 32 bit precision coefficients $\llbracket\left\{a \rrbracket_{k}\right\}$ for digital low pass Chebyshev type II filter from order 1 to 7 respectively. Figure 3(n) indicates that the filter obtained is unstable for high order less precision.

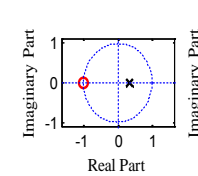

Real Part
(a)
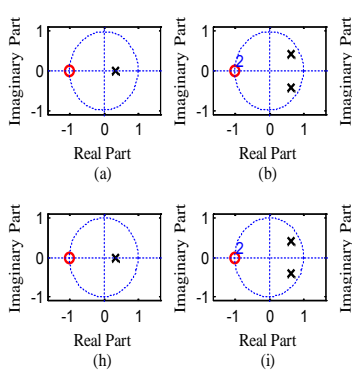

(b)
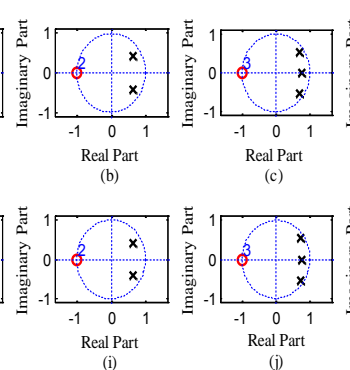

Real Part
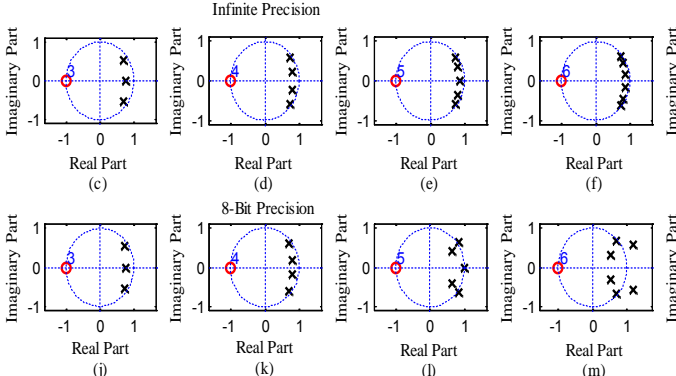

(e)
(eart
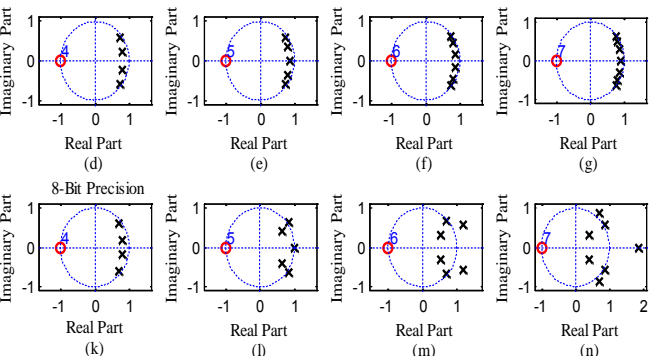

Real Part
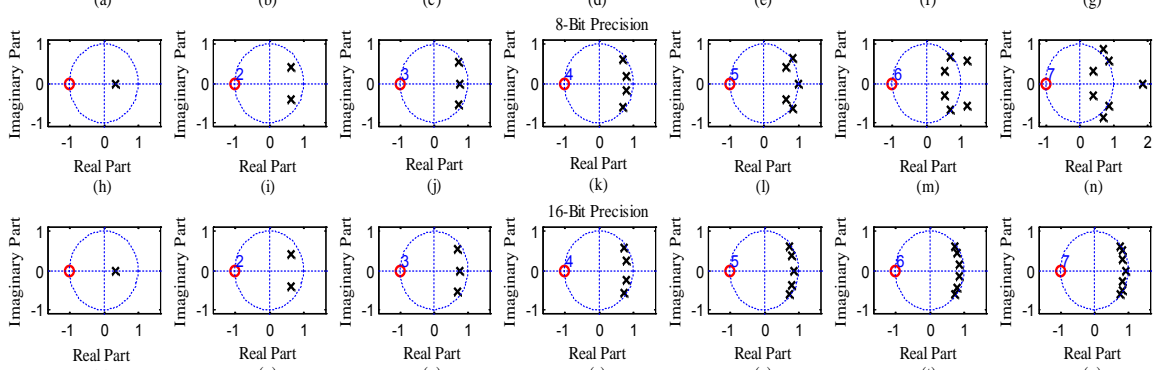

Real Part
$(\mathrm{s})$
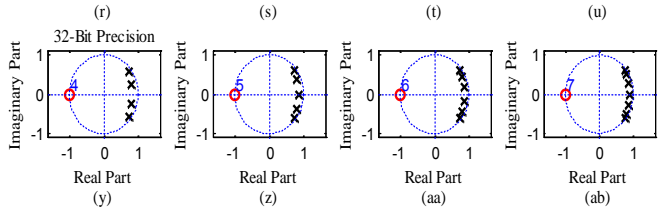

Figure 2. Pole and zero plot of digital chebyshev type i filter for infinite, 8-bit, 16-bit and 32-bit precision, where red circle indicates the zeros and black cross indicates the poles of the filter. 

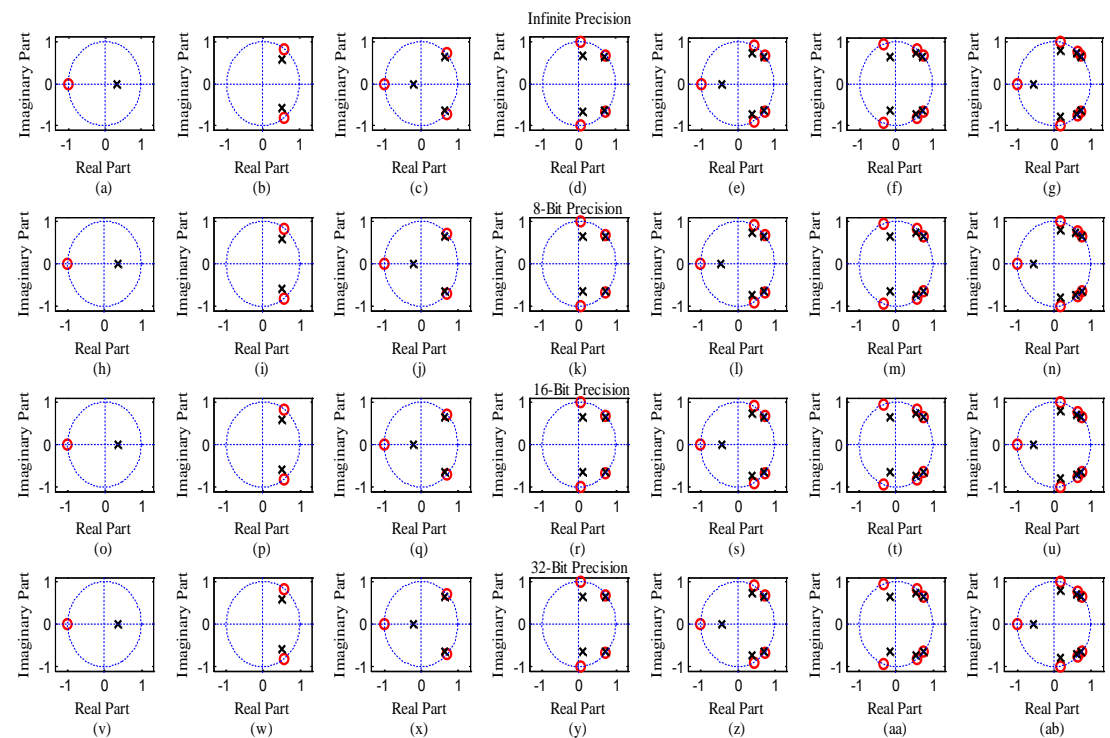

(e)
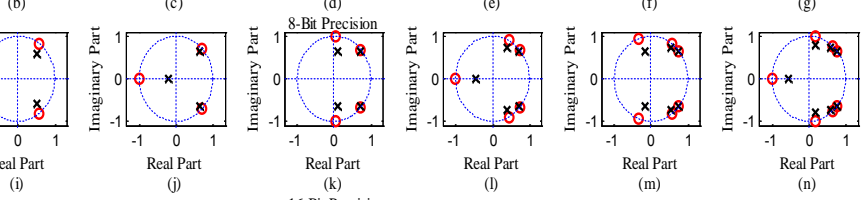

(j)
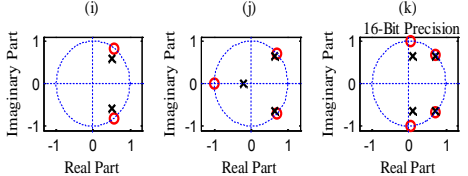

(I)
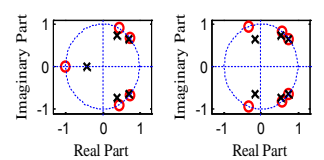

(n)
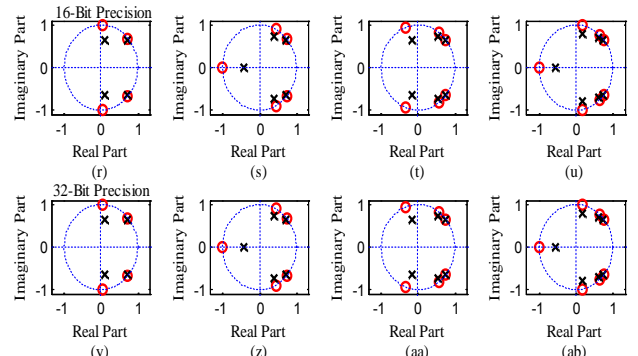

Figure 3. Pole and zero plot of digital chebyshev type ii filter for infinite, 8-bit, 16-bit and 32-bit precision, where red circle indicates the zeros and black cross indicates the poles of the filter.

Table 3. Perturbation error for digital chebyshev type II filter for 8-bit, 16-bit and 32-bit precision

\begin{tabular}{lccc}
\hline Order & 8-bit & 16-bit & 32-bit \\
\hline 1 & 0 & 0 & 0 \\
2 & $2.1691 \mathrm{e}-204$ & $1.0157 \mathrm{e}-206$ & $2.3236 \mathrm{e}-211$ \\
3 & $2.1091 \mathrm{e}-204$ & $1.4724 \mathrm{e}-206$ & $1.3118 \mathrm{e}-211$ \\
4 & $2.4246 \mathrm{e}-204$ & $5.5790 \mathrm{e}-207$ & $7.1262 \mathrm{e}-212$ \\
5 & $5.9412 \mathrm{e}-206$ & $9.3888 \mathrm{e}-208$ & $1.8022 \mathrm{e}-213$ \\
6 & $1.6469 \mathrm{e}-205$ & $6.1471 \mathrm{e}-208$ & $4.0479 \mathrm{e}-213$ \\
7 & $4.2324 \mathrm{e}-206$ & $1.4336 \mathrm{e}-208$ & $1.7116 \mathrm{e}-213$ \\
\hline
\end{tabular}

When $R=1 d B, W_{s t}=1$ and $n$ is the order that varies from 1 to 7 , the total perturbation error $\Delta p_{m}$ is calculated for the digital Chebyshev type II filter is as shown in Table 3.

\section{Digital Elliptic Filter}

The magnitude response of Elliptic filter exhibits equiripple characteristics in both pass band and the stop band The magnitude squared frequency response of analog low pass Elliptic filter is given as

$|H(j \omega)|^{2}=\frac{1}{1+\varepsilon^{2} J_{M}\left(\omega / \omega_{p}\right)}$

Where $I_{M}$ is a Jacobian elliptic function of order $M$ and $\varepsilon$ is parameter related to pass band ripple in Eq.
9. The inbuilt function $[b, a]=\operatorname{ellip}\left(n, R_{p}, R_{s}, W_{p}\right)$ using bilinear transformation converts the analog low pass Elliptic filter prototype into digital domain.

Figure 4 shows the pole-zero plots after quantization of digital Elliptic filter denominator coefficients $\mathbb{Z}\left\{a \rrbracket_{k}\right\}$ with infinite precision from Figure4(a) to Figure 4(g), 8-bit precision from Figure 4(h) to Figure 4(n), 16-bit precision from Figure $4(\mathrm{o})$ to Figure $4(\mathrm{u})$ and 32-bit precision coefficients from Figure 4(v) to Figure 4(ab). In Figure 4(k) to Figure 4(n), Figure 4(r) to 4(u) and Figure $4(\mathrm{ab})$, pole-zero plots shows that the filter becomes unstable for higher orders and less precisions.

When $R_{p}=1 d B, R_{s}=2 d B, W_{p}=1$ and $n$ is the order of the filter that varies from 1 to 7 , the perturbation error is calculated for each order and precisions is as shown in Table 4.

Table 4. Perturbation error for digital elliptic filter for 8-bit, 16-bit and 32-bit precision

\begin{tabular}{lccc}
\hline Order & 8-bit & 16-bit & 32-bit \\
\hline 1 & 0 & 0 & 0 \\
2 & $2.6096 \mathrm{e}-204$ & $4.1908 \mathrm{e}-207$ & $2.6196 \mathrm{e}-211$ \\
3 & $6.7338 \mathrm{e}-204$ & $7.1252 \mathrm{e}-206$ & $2.0993 \mathrm{e}-211$ \\
4 & $9.3245 \mathrm{e}-193$ & $3.355 \mathrm{e}-195$ & $1.1629 \mathrm{e}-199$ \\
5 & $5.7828 \mathrm{e}-163$ & $3.3989 \mathrm{e}-165$ & $3.5166 \mathrm{e}-170$ \\
6 & $2.6478 \mathrm{e}-84$ & $5.5627 \mathrm{e}-86$ & $1.2764 \mathrm{e}-90$ \\
7 & $1.2832 \mathrm{e}+47$ & $7.4982 \mathrm{e}+44$ & $6.5229 \mathrm{e}+39$ \\
\hline
\end{tabular}




\section{Comparison}

Table 5 shows the average perturbation error of each IIR filter for 8-bit, 16-bit and 32-bit precisions.From Table 5 it is clear that perturbation error decreases as bit precision increases from order 2 to 7 for all the digital IIR filter above described.

Table 5. Average perturbation error for digital IIR filters for 8-bit, 16-bit and 32-bit precision

\begin{tabular}{lccc}
\hline Filter & 8-bit & 16-bit & 32-bit \\
\hline Butterworth & $2.2689 \mathrm{e}-115$ & $4.2076 \mathrm{e}-120$ & $9.0067 \mathrm{e}-123$ \\
Chebyshev Type I & $8.4941 \mathrm{e}-144$ & $2.6418 \mathrm{e}-147$ & $1.8748 \mathrm{e}-151$ \\
Chebyshev Type II & $9.9560 \mathrm{e}-205$ & $4.5938 \mathrm{e}-207$ & $6.3195 \mathrm{e}-212$ \\
Elliptic & $1.8331 \mathrm{e}+46$ & $1.0712 \mathrm{e}+44$ & $9.3184 \mathrm{e}+38$ \\
\hline
\end{tabular}

The perturbation error is least in Chebyshev Type II filter and digital Butterworth filter have the highest perturbation error for all bit precisions.Digital Chebyshev type I and digital Chebyshev type II filters have perturbation error in between the digital Butterworth and digital Elliptic filter. From Table 1, Table 2, Table 3 and Table 4, the perturbation error of digital IIR filters increases linearly except for digital Chebyshev type II filter. It is clear that from order 2 to 7 the perturbation error for Elliptic filter increases rapidly. Whereas the perturbation error for order 1 it comes out to be zero as from equation 3 it is clear that perturbation error is inversely proportional to the difference between the two pole lengths.

Results shows that for higher orders direct form realization of digital Elliptic filters the pole sensitivity problem comes out to be very high whereas for digital Chebyshev type II filter it is the least. Thus the direct form realization of digital Chebyshev type II filter proves to be efficient for higher orders than all other digital IIR filters described above and worse for the digital Elliptic filter. Also from Figure 1, Figure 2, Figure 3 and Figure 4 it is clear that the digital elliptic filter becomes unstable faster than the other digital IIR filters due to increase in pole sensitivity problem.

\section{Conclusion}

Perturbation error is related to order of the filter and the bit precision. Increase in bit precision decreases the perturbation error and increase in order increases the perturbation error. Bit precision also affects the stability of the filter. As the bit precision increases the stability of the filter also increases. The direct form realization for higher orders is not beneficial as it gives a lot of error. The digital Elliptic filter gives the highest perturbation error
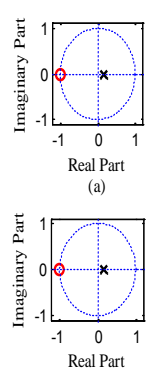

(h)

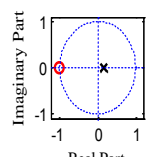

Real Part

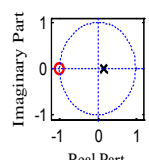

Real Pat
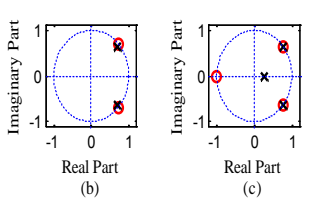

Real Part

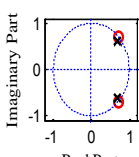

(i)

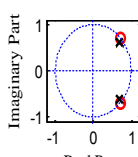

Real Part

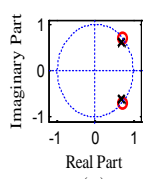

(w)

Real Part

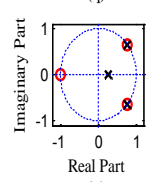

(x)

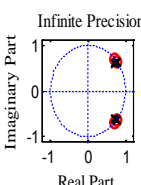

Real Pat

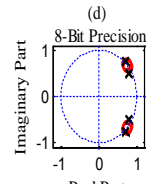

Real Part

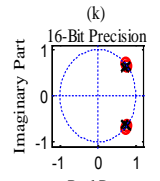

Real Part

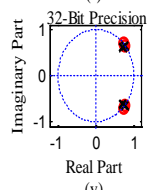

(y)

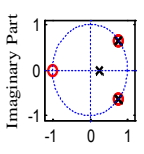

(e)

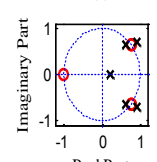

Real Part

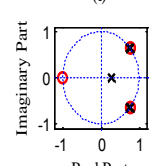

(s)
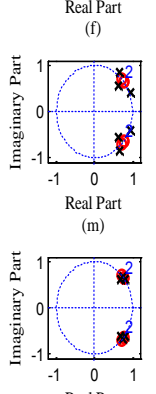

(t)

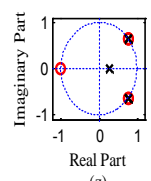

(z)

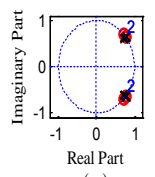

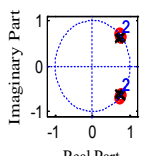

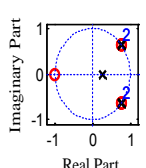

Real Part

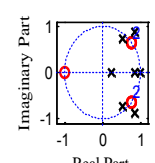

eal Pat

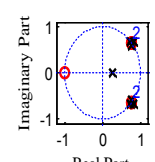

eal Pat

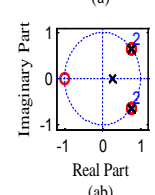

(ab)

Figure 4. Pole and zero plot of digital elliptic filter for infinite, 8-bit, 16-bit and 32-bit precision, where red circle indicates the zeros and black cross indicates the poles of the filter. 
than all other digital filters mentioned above for order greater than 3, while Chebyshev type II filter gives the least perturbation error. Further the quantization effect can also be realized for other IIR analog filters for cascade and other realizations.

\section{Future Scope}

Filters of higher order are preferred over lower order in practical applications as the magnitude response of the filter approaches towards the ideal magnitude response of the filter hence they effectively discriminate between the signals at different frequencies. Therefore they can be used in biomedical applications where two different signals needs to be discriminate like heart beat signals from the signals generated due to respiratory process. Also it finds application in cellular communication. IIR filters are generally used in applications where the phase distortion is tolerable. Filters with least perturbation error show least effect of finite word length and hence find application in the fields such as communication and biomedical science.

\section{References}

1. Proakis JG, Manolakis DG. Digital signal processing principles, algorithms, and applications. Chapter 9, section 9.5, Pearson Education Inc; 2007. p. 613-20.

2. Ingle VK, Proakis JG. Digital signal processing, A MATLAB based approach. Cengage Learning; 2008.

3. Dolecek GJ. Demo Program for Teaching the Characteristics of Low-Pass IIR Filters, $41^{\text {st }}$ ASEE/IEEE Frontiers in Education Conference, Session T4E, Rapid City, South Dakota; 2011 Oct. p. 1-6.

4. Allen HG, Perturbation effects on filters having multiple poles. Acoustics, Speech, and Signal Process. ICASSP-94, IEEE International Conference on Acoustics, Speech, and Signal Processing, Adelaide, SA. 1994 Apr 19-22; iii:58992.

5. Paarmann LD. Design and analysis of analog filters. Kluwer Academic Publishers; 2001. 